\title{
Supporting Information for "Ring Origami: Snap-folding of Rings with Different Geometries"
}

\author{
Shuai $\mathrm{Wu}^{1}$, Liang Yue ${ }^{1}$, Yi Jin ${ }^{1}$, Xiaohao Sun $^{1}$, Cole Zemelka ${ }^{1}$, H. Jerry Qi ${ }^{1}$, and Ruike \\ Renee Zhao ${ }^{1}$
}

${ }^{1}$ Affiliation not available

July 7, 2021

\begin{abstract} are shown in this paper.

Rich media available at https://youtu.be/ntHjYtsZvOY

Rich media available at https://youtu.be/0idhqs02LRI

Rich media available at https://youtu.be/VCqEqjEB6VE

Rich media available at https://youtu.be/kJDa42mtQeo

Rich media available at https://youtu.be/Y_CkIB2dx6s

Rich media available at https://youtu.be/aqyIOnXZw1k
\end{abstract}

Through experiment and simulation, we study a kind of deployable thin circular ring that can be folded into three small loops. Parametric study is conducted on the ring to study the geometric parameters that can influence ring's buckling .We also explore the rings with other shapes, such as elliptical rings, rectangular rings. Some applications basing on these studies 


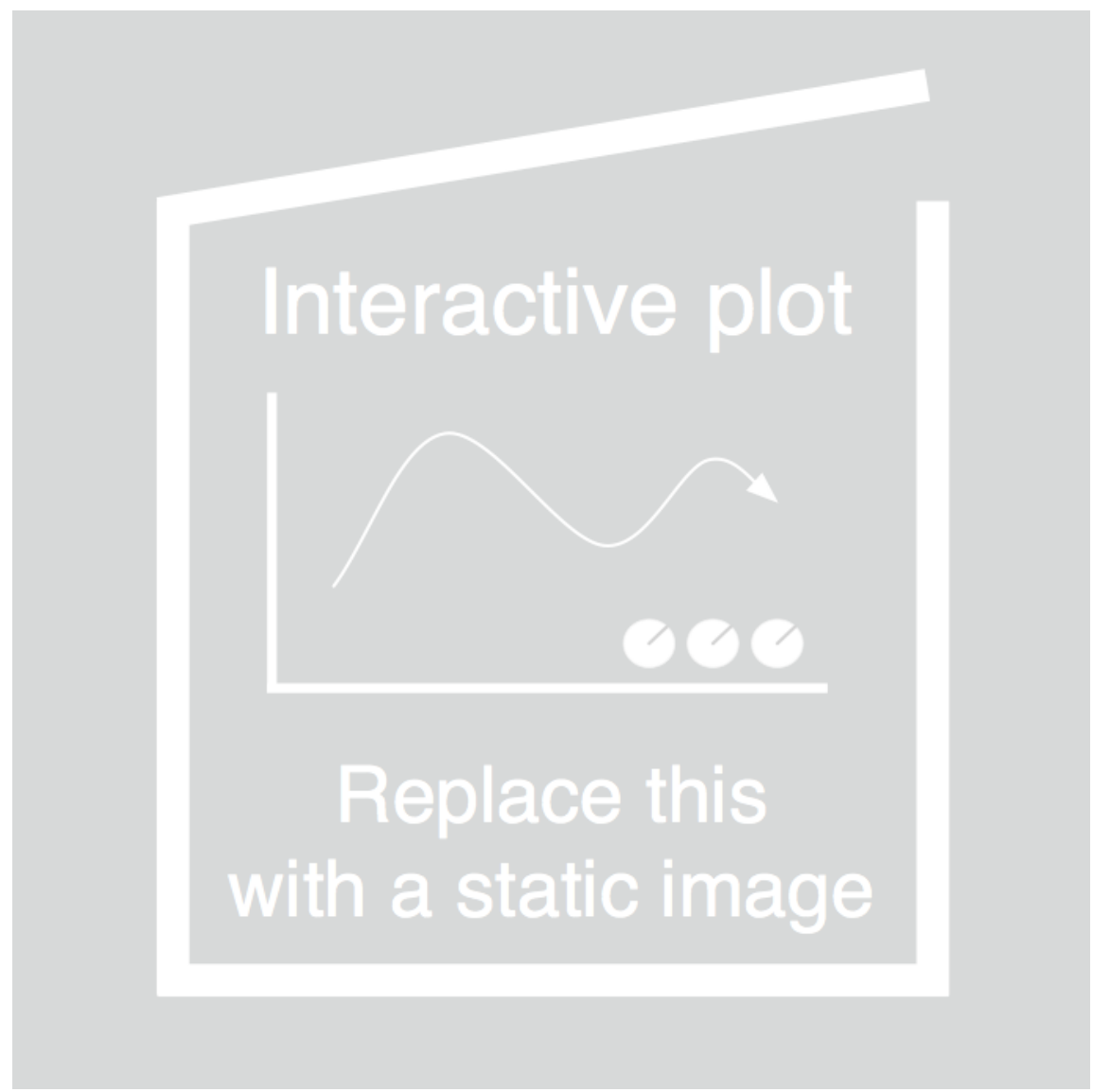

Figure 1: Snapping point of foldable circular ring 


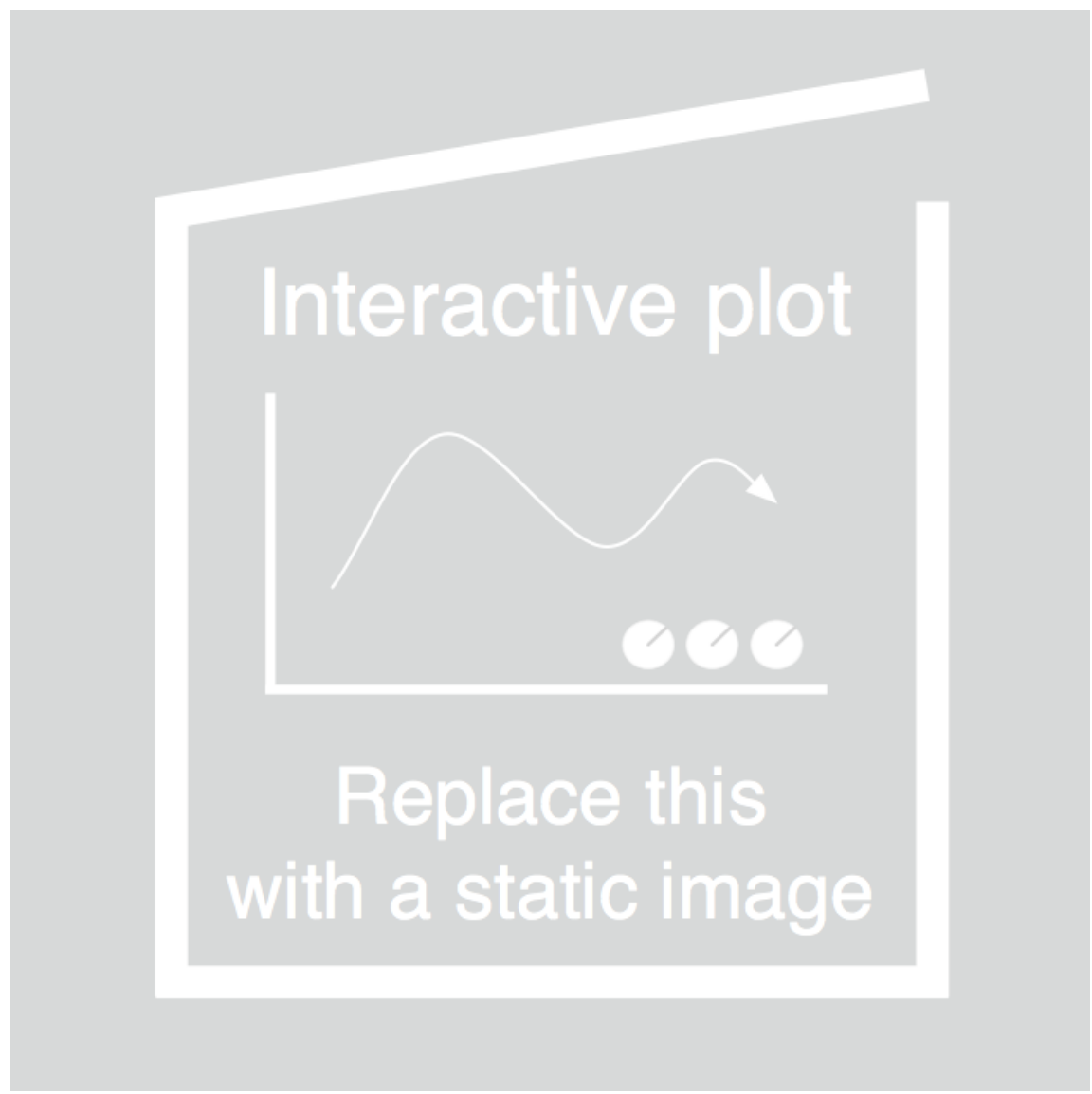

Figure 2: Final state of foldable circular ring 


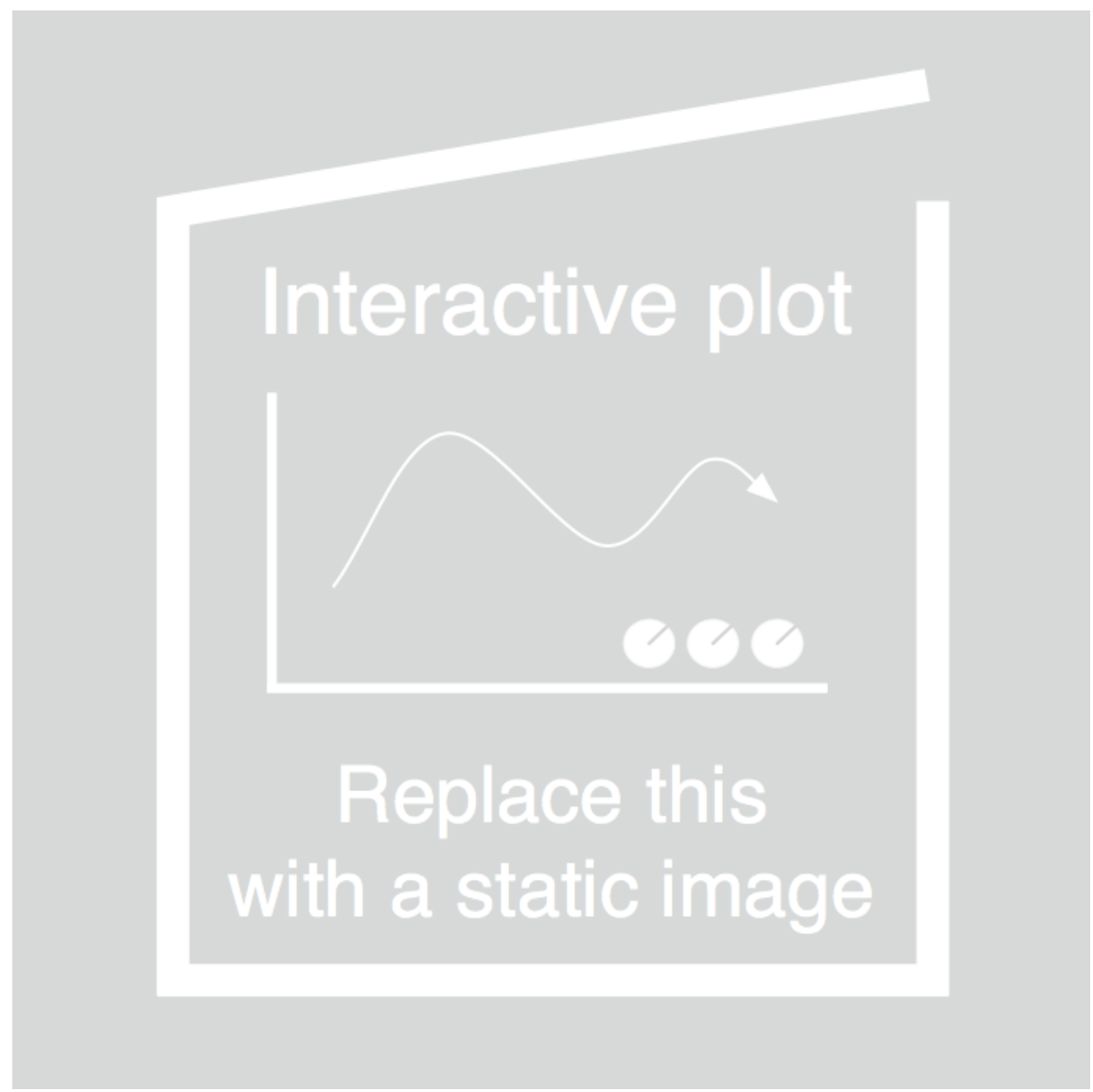

Figure 3: Snapping point of foldable elliptical ring 


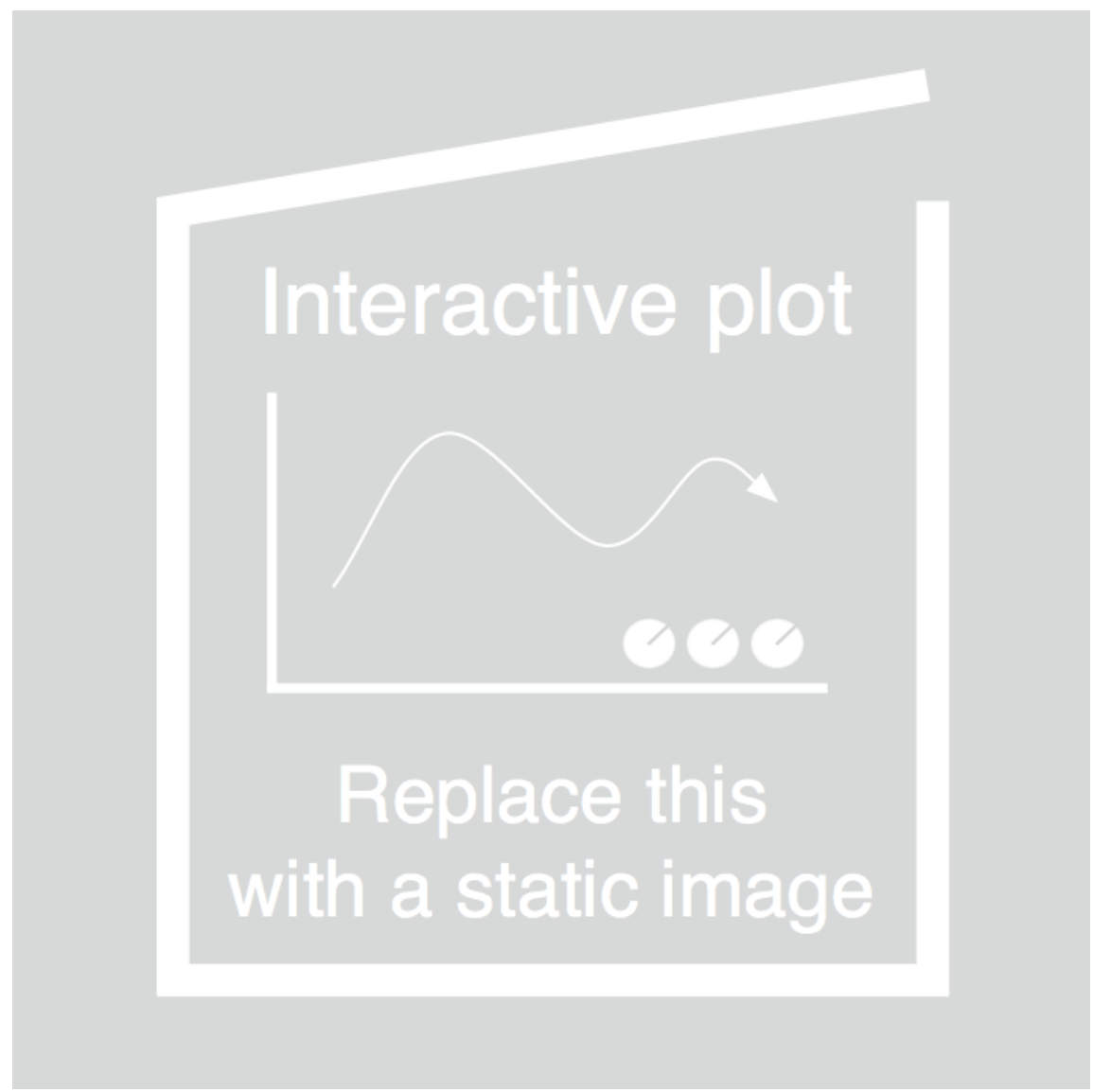

Figure 4: Final state of foldable elliptical ring 


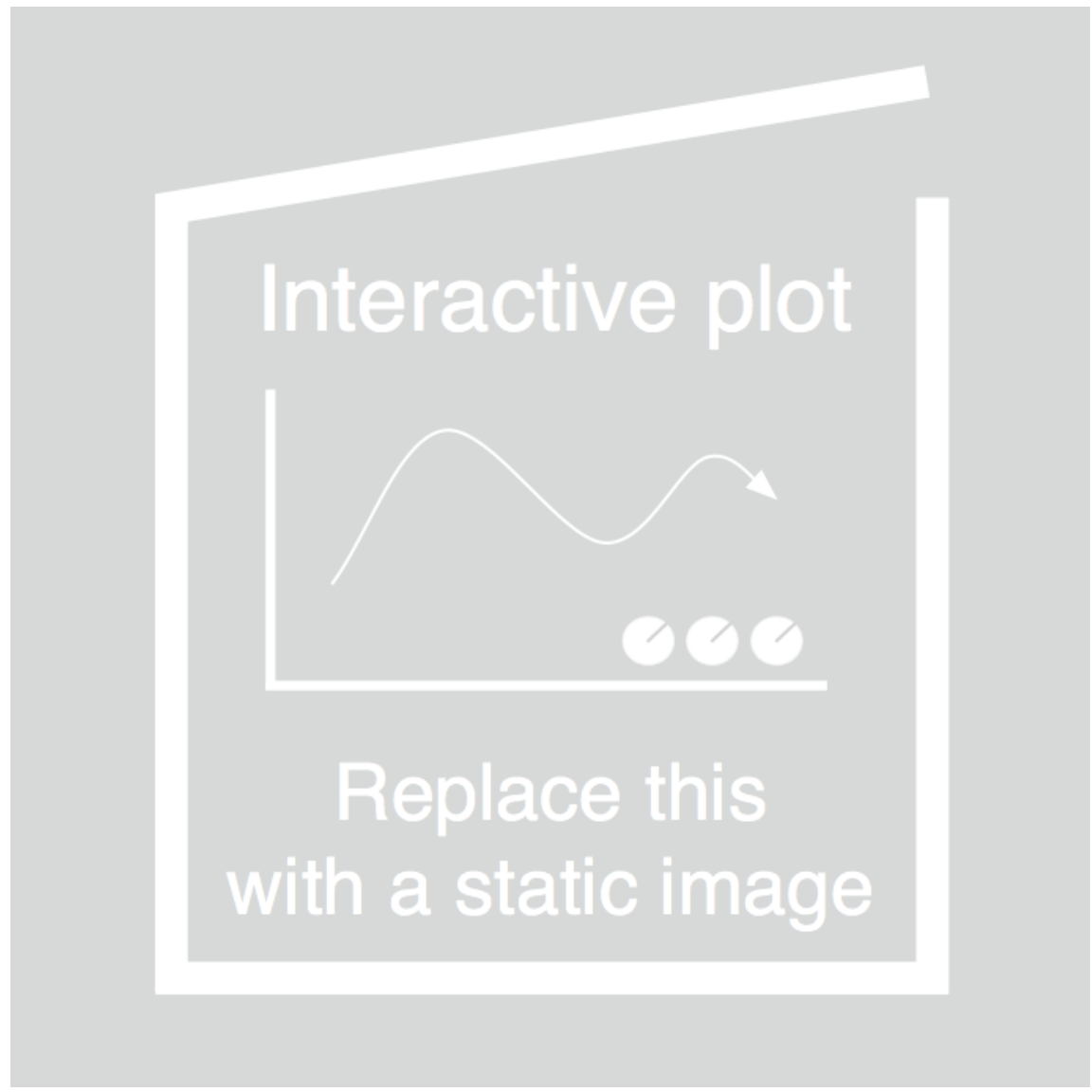

Figure 5: Snapping point of foldable rounded rectangular ring 


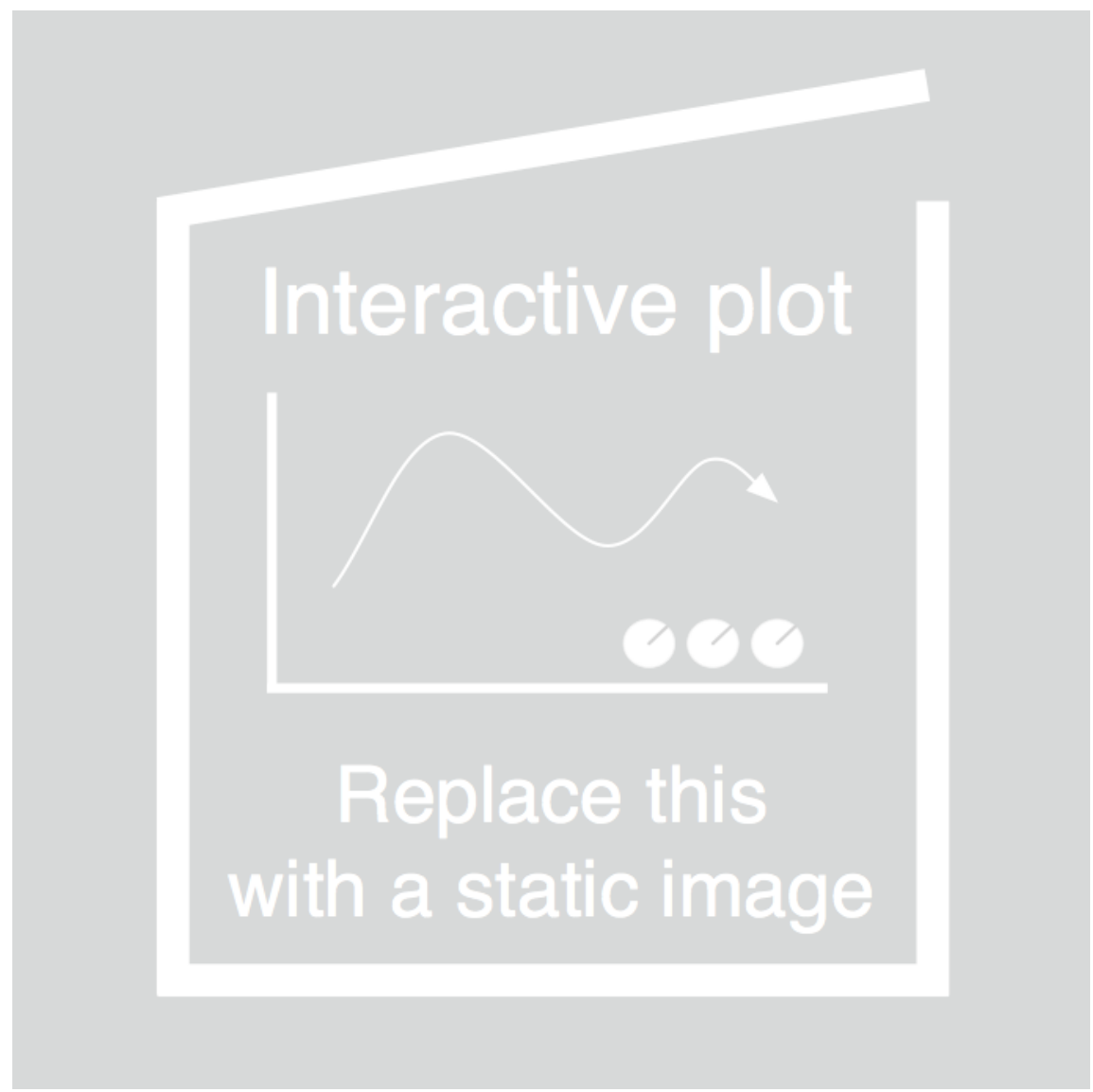

Figure 6: Final state of foldable rounded rectangular ring 


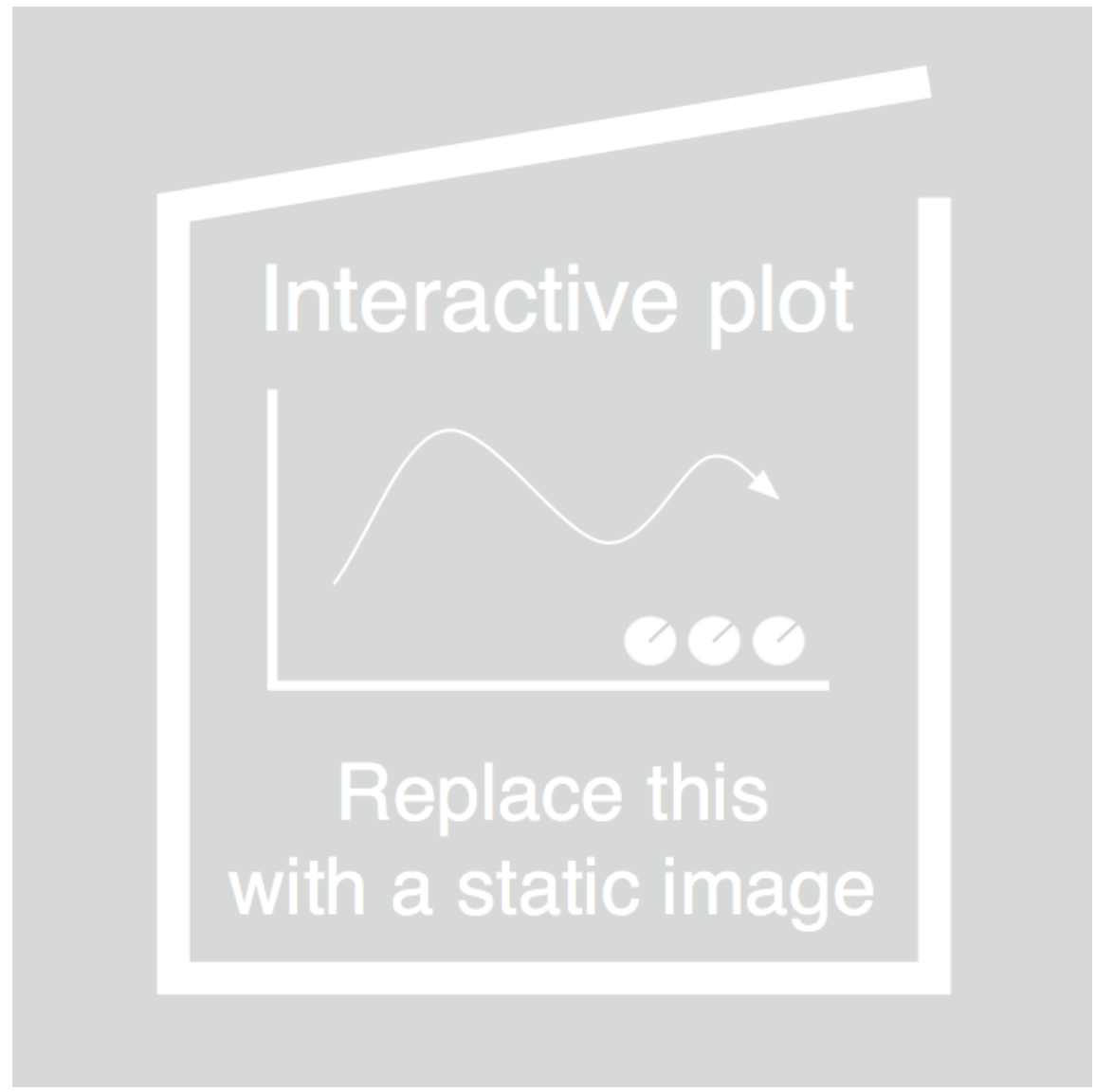

Figure 7: Snapping point of foldable rounded triangular ring 


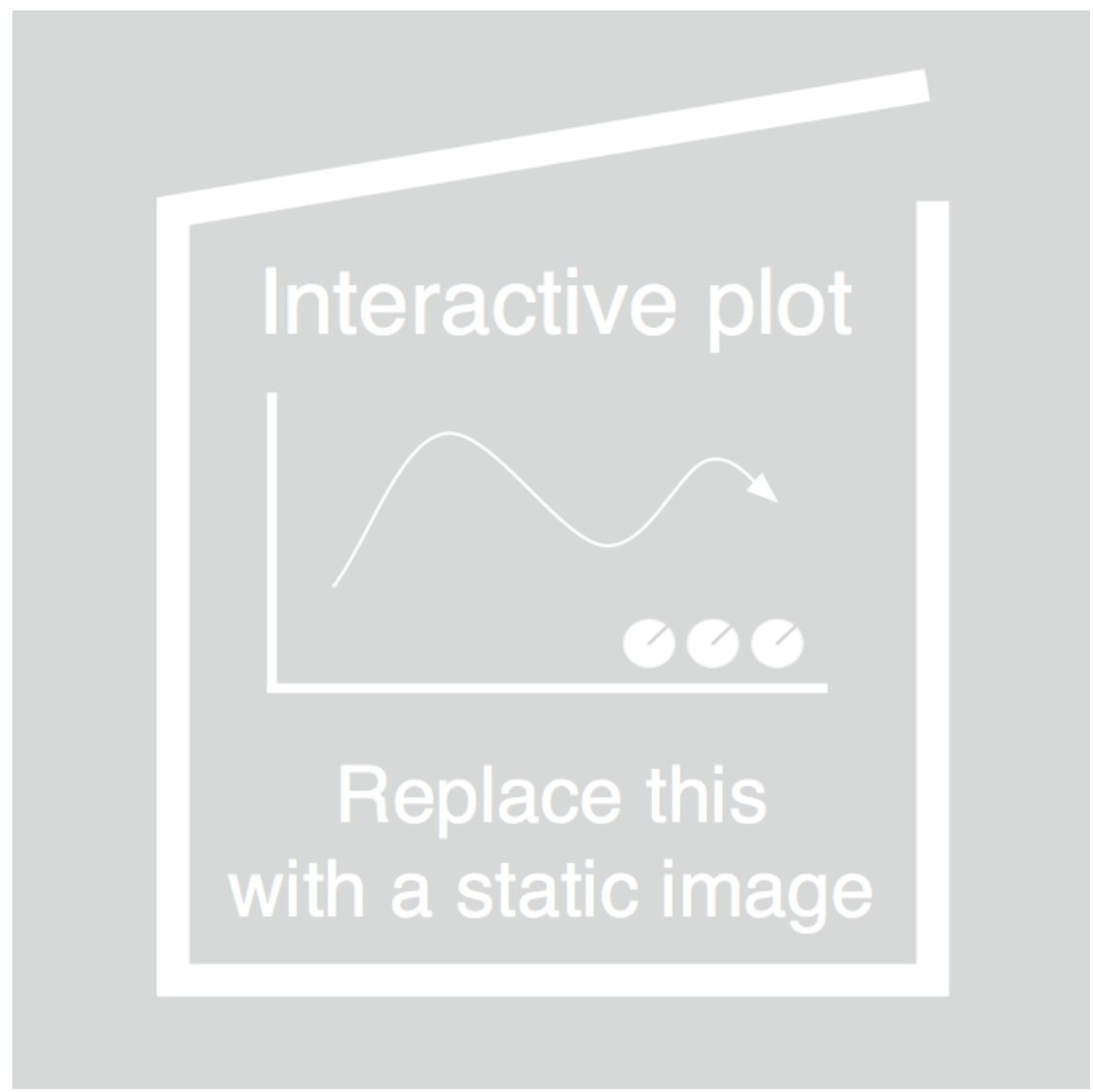

Figure 8: Final state of foldable rounded traingular ring 